\title{
DÜBLIN
}

Technological University Dublin

ARROW@TU Dublin

\section{Application of wavelet transform to power quality (PQ) disturbance analysis}

\author{
Malabika Basu \\ Technological University Dublin, mbasu@tudublin.ie \\ Biswajit Basu \\ Trinity College Dublin
}

Follow this and additional works at: https://arrow.tudublin.ie/engscheleart

Part of the Electrical and Computer Engineering Commons

\section{Recommended Citation}

Basu, Malabika and Basu, Biswajit :Application of wavelet transform to power quality (PQ) disturbance analysis. Proceedings of the Second International Conference on Power Electronics and Machine Drives (PEMD), Edinburgh, 31st. March-2 April 2004, pp.269-273. doi:10.21427/64qr-1d94

This Conference Paper is brought to you for free and open access by the School of Electrical and Electronic Engineering at ARROW@TU Dublin. It has been accepted for inclusion in Conference papers by an authorized administrator of ARROW@TU Dublin. For more information, please contact arrow.admin@tudublin.ie, aisling.coyne@tudublin.ie,gerard.connolly@tudublin.ie.

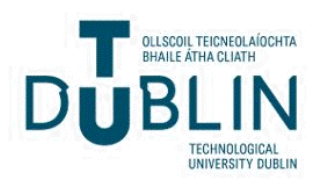




\title{
APPLICATION OF WAVELET TRANSFORM FOR POWER QUALITY (PQ) DISTURBANCE ANALYSIS
}

\author{
Malabika Basu*, Biswajit Basu ${ }^{\dagger}$ \\ *School of Control Systems and Electrical Engg \\ Dublin Institute of Technology, Ireland \\ email: mbasuldit.ie \\ † Dept of Civil, Structural and Environmantal Engg \\ Trinity College Dublin, Ireland \\ email: basubetcd.ie \\ Fax: $+3 \overline{53-1-6773072}$
}

Keywords: power quality, voltage sag, spike, flat-top, wavelet analysis, L-P basis

\begin{abstract}
This paper presents a wavelet analysis based time-frequency technique to identify power system transients and disturbances. A mother wavelet basis function (LittlewoodPaley (L-P)) with dyadic non-overlapping frequency bands has been used. The advantage of the proposed approach is in identification of the occurrence and duration of the disturbances. A few power signals with power quality disturbances have been analysed to show the effectiveness of the proposed technique.
\end{abstract}

\section{Introduction}

Power Quality (PQ) issues have been important for industrial facilities with sensitive loads. The transient events resulting in disturbances are necessary to be identified so that proper counter measures could be taken. Such counter measures are usually achieved by the use of power electronic apparatus. For some incidents, such as, caused due to a close by capacitor switching need not activate a Static Transfer Switch (STS) [1], while a voltage disturbance due to a remote fault may require a STS to operate. There are other incident events, e.g., a voltage sag/swell, which may require a Dynamic Voltage Restorer (DVR) to be operated to compensate the disturbance. Thus rapid detection of disturbances with identification of types of events is essential for PQ enhancement. Poor PQ may cause problems for the affected loads, such as malfunctions, instabilities, and short lifetime.

Poor PQ is usually caused by power line disturbances such as impulses, notches, glitches, momentary interruptions, over and under voltages, and harmonic distortions. In order to determine the causes and sources of the disturbances, it is not only required to detect and localise those disturbances but also identify and classify their types. Manual procedures for determining the disturbances are more expensive and inefficient in nature. The current state of the art with regard to detection of PQ disturbances is based on point-to-point comparison of adjacent cycles [2]. This technique suffers from the limitation that disturbances appearing periodically such as flat-topped and load controlled wave shape may not be detected. The approach of neural networks (NN) has been also used by [3] for the purpose of disturbance detcction. However, the approach based on NN is more specific as a particular type of $\mathrm{NN}$ is required to detect a particular kind of disturbance, and hence lacks generality in application.

The use of continuous wavelet transform (CWT) to analyse non-stationary harmonic distortion has been proposed by [45]. Further studies on $P Q$ assessment by using a dyadic orthonormal wavelet were carried out by [6]. Wavelets were used for analysing Electromagnetic transients by [7]. Other researches on $P Q$ detection and problems using wavelets include $[1,8,9]$. Corresponding to each scale the wavelet transform coefficients relate to the contribution of the signal at an instant of time and to that scale. This feature could be used to identify disturbances in a signal like sag and swell, transient electromagnetic disturbance, harmonic distortion etc. Most of the researches carried so far have used wavelet basis function, which have overlapping frequency bands. In this paper a wavelet basis function that has non-overlapping frequency band has been used. This would have the advantage of detecting disturbances corresponding to certain frequency bands, which are unaffected by the contributions from other bands. The wavelet basis function used is conventionally called Littlewood-Paley (L-P) basis function. This paper proposes techniques to detect and localise actual PQ disturbances measured by power line monitoring devices using L-P basis function. A scheme for classifying different types of $\mathrm{PQ}$ disturbances is also investigated using the wavelet analysis (WA) based time-frequency approach. A comparative analysis of the signals with FFT has also been reported to highlight the strengths of the wavelet analysis and the additional features it provides.

\section{Continuous Wavelet Transform}

Let $f(t)$ be a zero mean signal. The wavelet transform, $W_{\psi} f(a$, b) of this signal and the corresponding inversion relationship are given as follows [10] 


$$
W_{\psi} f(a, b)=\frac{1}{|a|^{1 / 2}} \int_{-\infty}^{\infty} f(t) \psi\left(\frac{t-b}{a}\right) d t
$$

and

$$
f(t)=\frac{1}{2 \pi C_{\psi}} \int_{-\infty}^{\infty} \int_{-\infty}^{\infty} \frac{1}{a^{2}} W_{\psi} f(a, b) \psi_{a, b}(t) d a d b
$$

with

$$
C_{\psi}=\int_{-\infty}^{\infty} \frac{|\psi \hat{(w)}|^{2}}{|w|} d w
$$

and

$$
\hat{\psi}(w)=\frac{1}{\sqrt{2 \pi}} \int_{-\infty}^{\infty} \psi(t) e^{-i w t} d t
$$

is the Fourier transform of the function $\psi(\mathrm{t})$. In Eq.(1), the function $\frac{1}{|a|^{1 / 2}} \psi((t-b) / a)$, often denoted by $\psi_{a, b}(t)$ (as in Eq.(2)) is obtained by translating the function, $\psi(t)$, to the time instant $t=b$ and then dilating by a factor of a. The function $\psi(\mathrm{t})$ is called the mother wavelet. The parameter, $b$, has the significance of localizing the basis function at $t=b$ and its neighbourhood. The parameter, $a$, captures the contribution of $f(t)$ to the frequency band corresponding to the Fourier transform of $\psi_{a, b}(t)\left(\right.$ i.e., $\left.\sqrt{a} \hat{\psi}(a w) \mathrm{e}^{-\mathrm{iwb}}\right)$. Thus, $W_{\psi} f(a, b)$ contributes to the function, $f(t)$, in the neighbourhood of $t=b$ and in the frequency band corresponding to the dilation factor $a$. This time-frequency localization feature of wavelet transform will be used for detecting PQ disturbances.

\subsection{Wavelet Basis function}

The basis function used as mother wavelet in this study is described by

$$
\psi(t)=\frac{1}{\pi \sqrt{(\sigma-1)}} \cdot \frac{\operatorname{Sin} \sigma \pi t-\operatorname{Sin} \pi t}{t}
$$

The Fourier domain characteristics of mother wavelet is given by

$$
\begin{aligned}
\psi(w) & =\frac{1}{\sqrt{2(\sigma-1) \pi}}, \pi \leq|w| \leq \sigma \pi \\
& =0, \text { otherwise }
\end{aligned}
$$

In particular, a value of $\sigma=2$ leads to L-P basis and harmonic wavelet proposed and used by $[11,12]$. A value of $\sigma=2$ has been used in this paper. The parameters $a$ and $b$ are continuous for CWT. For numerical computation of wavelet coefficients (WC) these parameters may be discretized for numerical calculation as, $a_{j}=\sigma^{j}$ and $b_{j}=(j-1) . \Delta b$, where, $i, j \in I$. Note that this discretization leads to a discrete parameter wavelet transform [13] of the CWT. Suitable choice of parameters $\sigma$ and $\Delta b$ will lead to orthogonal basis.

\section{Detection and Characterisation of PQ Disturbances}

In this section we apply and validate the proposed detection and localisation technique using some simulated $P Q$ disturbances in voltage signal of rms magnitude of $230 \mathrm{~V}$ and fundamental frequency $50 \mathrm{~Hz}$. These disturbances were simulated to represent the following cases

- Short duration harmonic distortion

- Voltage sag for a few cycles

- Flat topped waveform due to saturation

- Spikes in the voltage waveform, due to other network switching events

In order to detect a disturbance in a harmonic signal a timefrequency analysis using wavelets is performed. The magnitude of the CWT is calculated considering a few frequency bands. Since the fundamental frequency considered in all the cases is $50 \mathrm{~Hz}$, the fundamental frequency band for the mother wavelet is considered as $45-90 \mathrm{~Hz}$ ( $1^{\text {st }}$ band). Since a dyadic wavelet has been used, the six higher bands correspond to frequency ranges $90-180 \mathrm{~Hz}$ ( $2^{\text {nd }}$ band), 180 $360 \mathrm{~Hz}$ ( $3^{\text {rd }}$ Band), $360-720 \mathrm{~Hz}$ ( $4^{\text {th }}$ Band $), 720-1440 \mathrm{~Hz}\left(5^{\text {th }}\right.$ Band), $1440-2880 \mathrm{~Hz}$ ( $6^{\text {th }}$ Band) and $2880-5760 \mathrm{~Hz}\left(7^{\text {th }}\right.$ Band). WC are calculated by discretising the parameter $b$ at an interval of, $\Delta b=39.0625 \mu \mathrm{sec}(0.02 / 512)$. The calculated WC are used to identify and detect different kinds of disturbances. WC with high values indicates PQ disturbances, while smaller values are indicative of electrical noise.

\subsection{Characterisation of voltage sag}

When a voltage sag is detected in a signal, further computation to characterise the disturbance is necessary. The parameters of interest are magnitude, duration and shape of voltage sag. Also time and frequency location of the event is to be found. Fast detection of the instant of occurrence of sag will help in implementing fast and appropriate counter measures and would be useful for the protection of voltage sensitive loads. Fig. 1a shows a case of voltage signal of 5 cycles with a sag in voltage between $0.03-0.07 \mathrm{sec}$. The drop in voltage magnitude is $20 \%$. WC were calculated. The coefficients beyond $4^{\text {th }}$ band showed 2 spikes (leaving the initial transients) in voltage coefficients at 0.03 and $0.07 \mathrm{sec}$ correspond to the start and end of voltage sag. Fig lb shows a plot of WC of band $6(1440-2880 \mathrm{~Hz})$, where, the two spikes in WC indicating the start and end of the sag is very well manifested. In between the two spikes, the WC are very low. The application of FFT on the signal would also extract the peak amplitude reduction. But the exact instant and the duration of the voltage sag would be revealed by the WC only. 

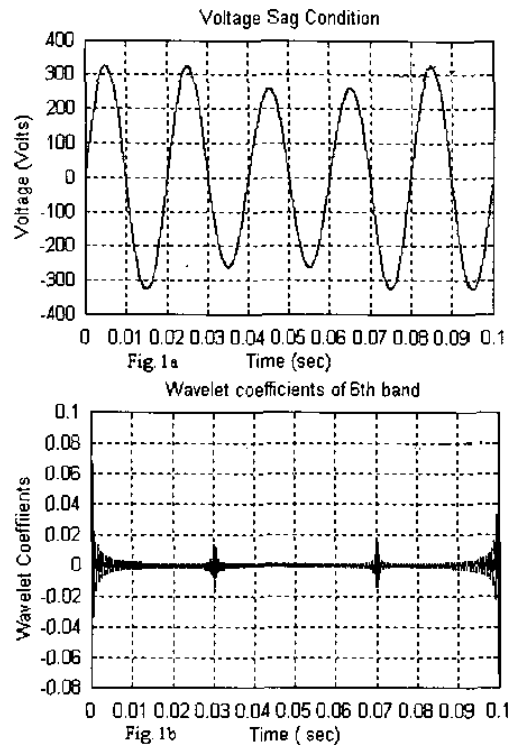

Fig 1a. Voltage sag (duration0.03-0.07 sec) detection with wavelets

Fig lb Wavelet coefficients of $6^{\text {th }}$ band
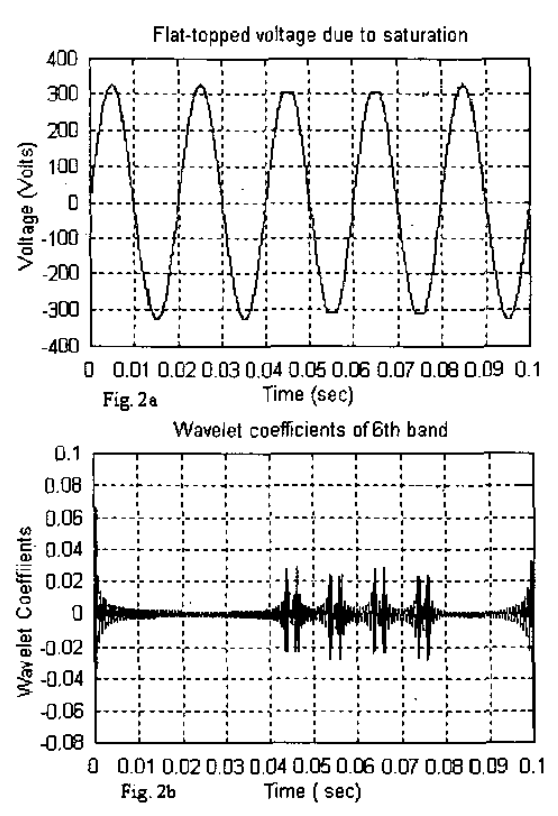

Fig. 2a Voltage signal with Flat-top problem Fig. 2b Wavelet coefficients of $6^{\text {th }}$ band

\subsection{Characterisation of saturation flat-topped voltage}

Fig. 2a shows an example case of a flat-topped waveform that may occur due to saturation phenomenon. This has been shown between $3^{\text {rd }}$ and $4^{\text {th }}$ power cycle. WC have been computed. The representation of WC in the $6^{\text {th }}$ band has detected these events accurately (Fig. $2 b$ ). There are multiple spikes present corresponding to the duration where saturation occurred. Four events have been detected with high spikes corresponding to the starting and ending time of saturation. In between the WC are moderately high, where as the coefficients are moderately low in the normal range of waveform. The results of FFT analysis plotted in Fig. 3 shows the presence of $3^{\text {rd }}, 5^{\text {th }}$ and $7^{\text {th }}$ harmonics apart from the fundamental one. The amplitudes of the $3^{\text {rd }} 5^{\text {th }}$ and $7^{\text {th }}$ harmonics are $1.6 \%, 1.4 \%$ and $0.9 \%$ of the fundamental respectively. The exact duration of flat-top is given by the increase in WC at the starting and finishing edge of flat-top. Similar information on WC were found when instead of five just one cycle of input signal with flat-topped characteristics was subjected to wavelet analysis. A comparison of the WC of $6^{\text {th }}$ band is shown in Fig. 4, where Fig. 4a shows the

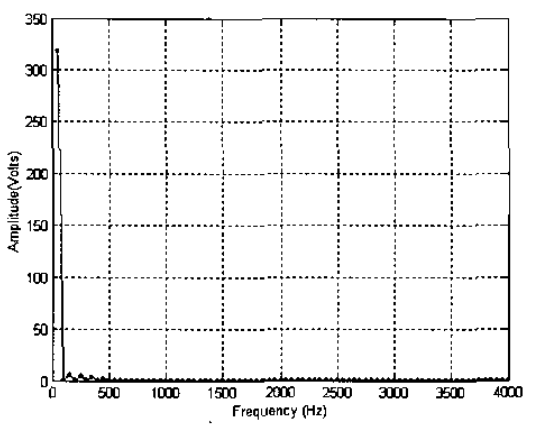

Fig. 3 FFT of Flat-top wave form shown in Fig. 2a

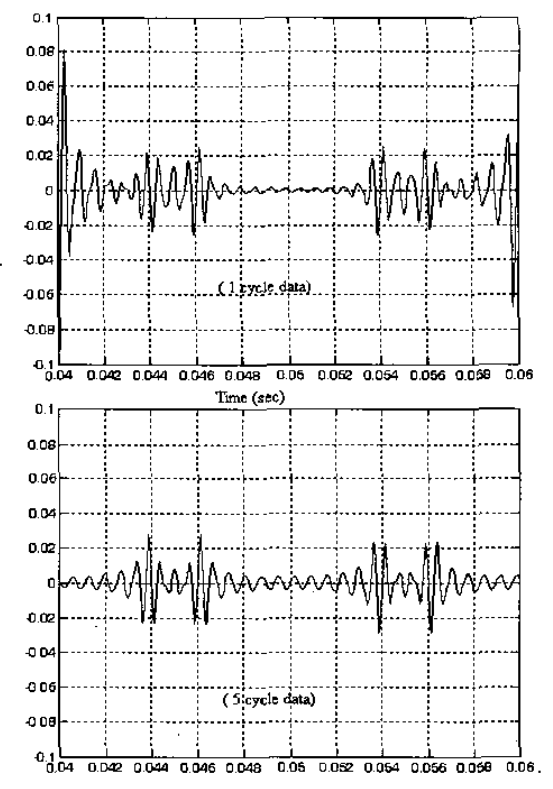

Fig. 4a Wavelet coefficients based on 1 cycle data of flat-top waveform shown in Fig. 2a

Fig. $4 \mathrm{~b}$ Wavelet coefficients based on 5 cycle data of flat-top waveform shown in Fig.2a 
coefficients of one cycle data and Fig. $4 b$ show the WC of 5 cycle data. As seen from the figure, the values of the $\mathrm{WC}$ are very much close to each other. Therefore, significant data compression is possible. This could be an advantageous feature in PQ data monitoring for analysis and storage.

\subsection{Characterisation of high frequency harmonic distortion}

Fig 5a shows five cycles of power signals with fundamental frequency $50 \mathrm{~Hz}$. In the $3^{\text {rd }}$ and $4^{\text {th }}$ cycle the nature of the wave shape have been distorted due to high frequency decaying noise. The noise signal, $\mathrm{S}(\mathrm{t})$ has been simulated by the following Eq. 7 in the appropriate small interval $[0.004$ sec]

$50 \sin (30.2 \pi 50 \mathrm{t}) \cdot \exp (-20 \mathrm{t})$

The WC corresponding to band 6 again captures this as shown in Fig. 5b. WC have been found to be uniformly high over these phases where the distortions are present.
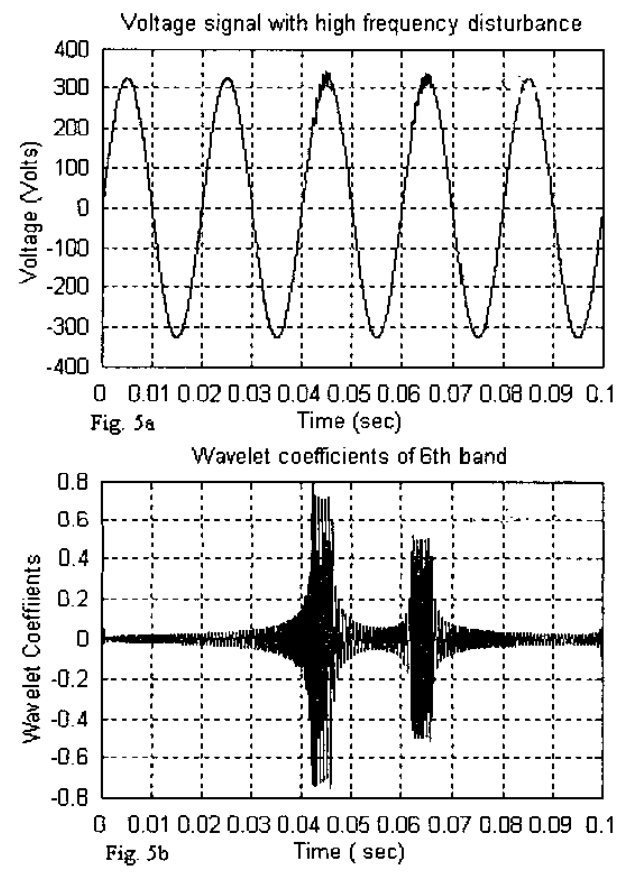

Fig.5a Voltage signal with high frequency distortion Fig. $5 \mathrm{~b}$ Wavelet coefficients of the $6^{\text {th }}$ band

Fig. 6 shows FFT of the voltage signal with high frequency distortion. A high frequency harmonic $(1500 \mathrm{~Hz})$ has been detected in the FFT. However, the exact time of occurrence is revealed only by WC which could be useful in finding out the cause of such high frequency distortion. This is an added advantage of using wavelet analysis as compared to the Fourier based analysis.

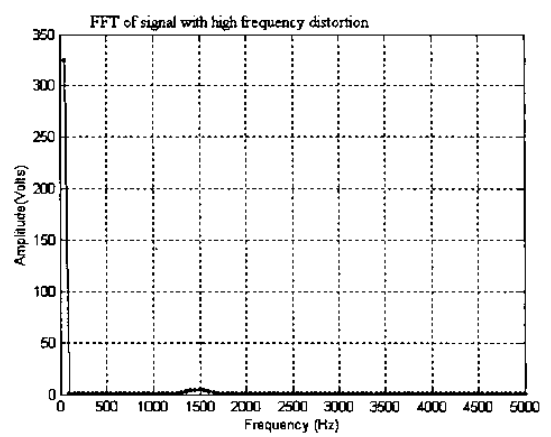

Fig. 6 FFT of signal with high frequency distortion

\subsection{Characterisation of voltage spike}

Fig. 7a shows a voltage waveform signal with 2 sharp spikes over very short duration in the $3^{\text {rd }}$ and $4^{\text {th }}$ cycle respectively. WC plotted in Fig. $7 \mathrm{~b}$ is characterised by two sharp spikes, corresponding to the location of input spikes. Rest of the coefficients are quite low.
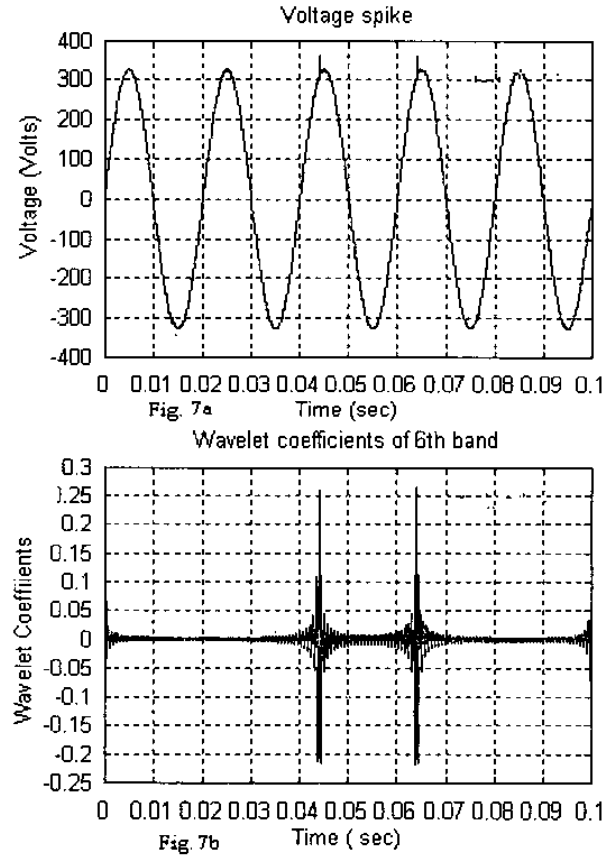

Fig. 7a Voltage signal with high frequency spike Fig. 7b Wavelet coefficients of the $6^{\text {th }}$ band

On comparing the results of FFT analysis on the signal with spike, it is interesting to note that FFT cannot detect the presence of high frequency spikes at all, as their energy content is very low, but WC could extract out the phenomenon very accurately. 


\section{Conclusion}

An efficient yet simple technique has been developed to detect and characterise PQ disturbances. This approach very accurately locates voltage sag, spike, harmonic distortion and voltage saturation temporally. The results show accurate classification of events can be performed by wavelet based time-frequency characteristics. Unlike Short Term Fourier Transform, the analysis is not window dependent and numerically efficient. This method can be extended to detect other PQ events that need to be monitored. Further, this could be utilised for achieving on-line PQ monitoring.

\section{References}

[1.] [1] M. Karimi, et al, "Wavelet based on-line disturbance detection for power quality applications," IEEE Trans. on Power Delivery, vol.15, no. 4, pp. 1212-1220, Oct. 2000.

[2.] McEachern, Handbook of Power Signatures. Basic Measuring Instruments, Foster City, CA, 1988.

[3.] N. Kandil, V. K. Sood, K. Khorasani, and R. V. patel, "Fault identification in an AC-DC transmission system using neural networks," IEEE Trans. on Power Systems, vol. 7, no. 2, pp. 812-819, May, 1992.

[4.] S. santoso, et al, "Electric power quality disturbance detection using wavelet transform analysis," in Proceedings of the IEEE-SP International Symposium on Time-Frequency and Time-Scale Analysis, Philadelphia, PA, Oct. 25-28, 1994, pp. 166-169.

[5.] P. F. Ribeiro, "Wavelet transform: an advanced tool for analysing non-stationary harmonic distortions in power systems," in Proceedings of the IEEE International Conference on Harmonics in Power Syetms, Bologna, Italy, September 1994.

[6.] S. Santoso, et al, "Power quality assessment via wavelet transform analysis," IEEE Trans. on Power Delivery, vol.11, no. 2, pp. 924-930, April 1996.

[7.] D. C. Robertson et al, "Wavelets and electromagnetic power system transients," IEEE Trans. on Power Delivery, vol.11, no. 2, pp. 1050-1056, April 1996.

[8.] G. T. Heydt, and A. W. Galli, "Transient power quality problems analysed using wavelets," IEEE Trans. on Power Delivery, vol.12, no. 2, pp. 908-915, April 1997.

[9.] O. Poisson, et al , "Detection and measurement of power quality disturbances using wavelet transform," IEEE Trans. on Power Delivery, vol.15, no. 3, pp. 1039-1044, July 2000.

[10.] I. Daubechies, "An introduction to wavelets," San Diego, Calif., U.S.A., Academic press, 1992.

[11.] B. Basu et al, "Seismic response of SDOF systems by wavelet modelling of nonstationary processes", Amer. Soc. Civ. Eng., J. Eng. Mech., 124(10), 1998, pp.11421150.

[12.] D. E. Newland, "An introduction to random vibrations, spectral and wavelet analysis", Longman, U.K. 1993.

[13.] Y. T. Chan, "Wavelet Basics", Kluwer Academic Publishers, MA, 1995. 